\section{Communication and Learning in the New Media Space}

\begin{abstract}
Gábor Szécsi*
Abstract: The age of electronic communication is the age of opening categorical and classification boundaries. In the new media space the traditional distinctions between children and adult experiences collapse and disappear. The aim of this essay is to show that the use of electronic technologies has abolished the traditional pedagogical thinking, and brings in new conventions. As a result of evolving new practices which rely on electronic communication devices, communication has become an essential activity among children, helping them acquire and share everyday information and knowledge with intensity and efficiency that can even change the traditional pedagogical thinking. The use of new communication technologies and forms of learning support gain particular importance especially in a system of lifelong learning, which provides identical frameworks for children and adults.
\end{abstract}

Keywords: electronic communication technologies, networked individual, disappearance of childhood, new pedagogical paradigm.

By using the electronic communication technologies the media-networked individuals become members of a virtual community that is determined by the global and local conditions for an effective method of information exchange. In this new, virtual community the traditional distinctions between children and adult experiences collapse and disappear. This paper argues that the new perspective created by the electronic communication has transformed not only our concept of learning and the traditional role of a teacher but also our notion of pedagogy as a science. Electronic communication has become an essential activity among the young, helping them acquire and share everyday information and knowledge with an efficiency that can even crack the conservative shell of pedagogical thinking. I will attempt to show that the expansion of electronic communication technologies, therefore, leads us to a new way of thinking about learning and teaching.

\section{A networked individual in electronic societies}

Linguistic communication is creating an action of a society, community, and an individual. It is a creative process which determines and forms our personality and identity through our communication roles. Moreover, the human mind is a structure affected by the prevailing technologies of social communication. That is to say, there is a specific inner relation between the communicative structure of our minds and the communication technologies which can be regarded as dominant in certain cultures.

The inner relation is present in all major historical changes in dominant communicative technologies, from the change-over to the literacy with the dominance of electronic media (television, the internet and mobile telephone). In this essay, I try to prove the above-mentioned assumptions of the effects of electronic media on our conceptualization of community.

\footnotetext{
* Gábor Szécsi, Institute for Philosophical Research of the Hungarian Academy of Sciences, Hungary; szecsi@webmail.phil-inst.hu

Department of Cultural Mediation, Faculty of Adult Education and Human Resources Development, University of Pécs, Hungary; szecsi@feek.pte.hu
} 


\section{Acta Technologica Dubnicae \\ volume 2, 2012, issue 1}

To clarify the nature of this new conceptualization, I take the hypothesis as a starting point - the twentieth-century expansion of electronic communication has transformed our notion of the relation between a place and a community. With a greater proportion of our communicative acts taking place via electronic media, the physical co-presence and the co-located interpersonal relations, as the determinants of the nature of human interactions, are diminishing.

It seems that in the space of electronic media, a community should be understood as a virtual network of interactions between individuals who uniformly accept and apply some rules of communication aimed at the effective exchange of information. In other words, there is an inner relation between the criteria of community and the global and local conditions for an effective method of information exchange. The global and local conditions transform our notions of the structure and life of a community.

Electronic communication creates a new context in which our notions of culture, community, society and human interactions become more complex. These notions can be regarded as the bases of the idea of the global and local information communities in which the communication attitudes of people are determined by their impression of themselves as permanently available individuals whose communicative acts are embedded in special information net.

This paper argues that by creating new, information communities, the electronic technologies have been speeding up through the crossing of traditional conceptual, social, cultural and political boundaries. As a result of this process, we are experiencing conceptual, social and cultural convergences in new global and local forms of communities.

My general argument is that the bases of such conceptual, social and cultural convergences are the linguistic convergences which lead to the appearance of a new kind of communication language. With the interaction of global and local communicative situations of the electronic era, most linguistic, cultural and social boundaries have become more permeable. These permeable linguistic, cultural and social boundaries affect both the particular behavior and social identity. The increasing functional permeability of these boundaries is a result of such linguistic, cultural and social processes that contribute to develop the media-networked individuals' complex identity (Nyíri, 2005).

The complex identity, however, is rooted, first of all, in the new forms of communities. In other words, the use of electronic media can make our concept of community more complex by creating new kinds of communities. A networked individual becomes a member of both a global community based on the global communication crossing national cultural boundaries and a local community that is organized on the basis of specific, inner norms in the space of electronic communication.

As Joshua Meyrowitz (2005) writes about the "multiple, multi-layered, fluid, and endlessly adjustable senses" of the media-networked individuals' identity: "Rather than needing to choose between local, place-defined identities and more distant ones, we can have them all, not just in rapid sequence but in overlapping experiences. We can attend a local zoning board meeting, embodying the role of local concerned citizen, as we cruise the internet on a wireless-enabled laptop enacting other, non-local identities. And we can merge the two as we draw on distant information to inform the local board of how other communities handle similar issues and regulations. All the while, we can remain accessible to friends, family, and colleagues from anywhere via a text-message enabled mobile phone."

By using the electronic communication technologies, a networked individual becomes a part of a network of interactions between humans who uniformly accept and apply some rules for the communicative acts aiming at the effective exchange of information. In other words, the medianetworked individuals become members of a virtual community that is determined by both the global and the local conditions for an effective method of information exchange. 


\section{Acta Technologica Dubnicae \\ volume 2, 2012, issue 1}

In this new virtual community, new localities are being made which are particular in many ways and influenced by global processes and global consciousness. Thus the new local communities organized in the space of electronic communication, on the one hand, strengthen the local attachments, the local identity and, on the other hand, can be regarded as integrated elements of virtual communities created by global information exchange. Consequently, the global virtual community serves as a kind of comparison background for the local communities organized in the age of electronic media. With globalized communication space, electronic media give the networked individual external perspectives from which to judge and define one's own local community. In other words, the twentieth-century expansion of electronic communication technologies, as J. Meyrowitz (2005, p. 23) writes, "have placed an interconnected global matrix over local experience". The networked individual determines the characteristics of his own local community in the light of information acquired in the global communication space.

The global perspective created by electronic communication has transformed not only the community definitions but the individual relation to social rules. In the space of electronic communication there is a new possibility to change the rules of social perception and the national institutions of political and cultural domination as a consequence of new global perspectives.

\section{Towards a new way of thinking about learning and teaching}

One of the most characteristic features of the virtual space of electronic communication is that it lacks the compulsory categorization system and the classificatory forms and norms of a print society. In the media-networked global and local communities it is difficult to maintain several traditional categorical distinctions that characterized the print societies. That is, as electronic communication technologies expand, the dividing line between several political and social categories becomes increasingly indistinct.

The age of electronic communication is the age of opening categorical and classification boundaries. In this new space of communication the traditional distinctions between private and public, between children and adult experiences, and between male and female spheres collapse and disappear. In the age of electronic media, as Meyrowitz (2005, p. 29) suggests, we are experiencing "both macro-level homogenization of identities and micro-level fragmentation of them".

A new virtual social space is in the progress which strengthens the cohesion of competing local communities, and in which, therefore, the influence of traditional social and political institutes declines. The new communication situations created by the use of electronic technologies foster greater emotional attachments to the local community which we choose from among the competing communities deliberately without social and political restrictions.

Thus in this new social space there is a fundamentally new possibility to change the rules of social perception and the conceptualization of the relation between the local communities and traditional political institutes of state. Thanks to the changes, the networked individual is attached to the place and position appointed by his own social class less and less. Through his multi-channel communicative acts he can become acquainted with more and more communal forms, ways of life, traditions and values, in the light of which he can choose more deliberately from among the competing local communities. And this more deliberate choice becomes a part of the more and more complex and multi-layered identity of the networked individual.

One of the most important consequences of the openness of the new communication situations created by the use of electronic technologies is that the boundaries between childhood and adulthood became blurred. In Nail Postman's (1994) terms, the "disappearance of childhood" is one of the convergences of social and cultural categories experienced in the new media space. By using electronic technologies children are routinely exposed to the so called "adult information". As J. Meyrowitz (2005, p. 29) writes, "just as there is a blurring of traditional distinctions between children's and adults' experiences 


\section{Acta Technologica Dubnicae \\ volume 2, 2012, issue 1}

/.../, so is there a breaking down of the traditional similarities among what people of the same age or same gender experience".

The use of the electronic technologies, therefore, has abolished the traditional pedagogical thinking, and brings in new conventions. As a result of introduction of new practices which rely on electronic communication devices, communication has become an essential activity among children, helping them acquire and share everyday information and knowledge with an intensity and efficiency that can even change the traditional pedagogical thinking. The use of new communication technologies and the involving forms of learning supports gain particular importance especially in a system of lifelong learning, which provides identical frameworks for children and adults. So there is no sense in making distinctions between the children's and adults' world, since new forms and technologies of learning can be developed for and applied to any age group.

The "disappearance of childhood", accordingly, is one of the consequences of conceptual convergences that are rooted in the increasing functional permeability of the boundaries between communication situations in the age of electronic media. Electronic communication unties us from the restrictions and limitations implied in traditional social classing and interactions. As J. Meyrowitz (2005, p. 30) points out: "Yet, with a wide array of electronic media, including the mobile phone, we are also liberated from the same bounded and confining experiences. We are free to choose our own networks for membership and our own level of engagement in each network. We are free, as well, to shape our degrees of connection to local space." The expansion of electronic communication has abolished classroom enclosure and erodes the existing pedagogical norm system by this functional permeability of situational boundaries. By appearing a new virtual society, the traditional role of the teacher becomes anachronistic in this way.

The novelty of the structure and function of knowledge acquired by electronic communication is a consequence that affects the bases of traditional pedagogical thinking. It is a problem which provides new scope for pedagogical researches. The effects of this problem appear more and more significant in social and cultural dimensions that force pedagogical thinking in a wider framework. The change has an expanding effect on the framework of the new, electronic forms of learning, adding new dimensions. The use of electronic communication technologies sets pedagogy a big methodological challenge.

Pedagogy becomes a part of the process characterizing the scientific life of information society leading to disappearance of traditional disciplinary boundaries. And this disciplinary openness can be regarded as a basis of a new pedagogical paradigm which helps us to form general theory of the cognitive background. The consequences of the new teaching/learning forms appearing in the new media space can help us work out a methodological conception that offers appropriate solutions to the new cultural challenges.

\section{References}

MEYROWITZ, J.: The Rise of Glocality. New Senses of Place and Identity in the Global Village. In K. Nyíri (ed.). Sense of Place. The Global and the Local in Mobile Communication. Vienna: Passagen Verlag, 2005, p. 28.

NYÍRI, K.: The Networked Mind. A speech given at the workshop "The mediated mind - rethinking representation". London: The Knowledge Lab, Institute of Education, University of London, 2005, p. 27-28.

POSTMAN, N.: The Disappearance of Childhood. New York: Vintage Books, 1994. 